\title{
Die Suche nach dem Glück in der deutschen Literatur. Zur Bedeutung der blauen Blume in Novalis' Heinrich von Ofterdingen
}

Simone Malaguti*

\begin{abstract}
Heinrich von Ofterdingen, written by Novalis, is imprinted in the history of the German literature just like Goethe's masterpieces Die Leiden des jungen Werthers and Wilhelm Meisters Lehrjahre. It is a meaningful novel also because of its blue flower, a motive added by Novalis into his novel. This paper analyses this blue flower as a symbol of the search for happiness in the German literature. For this purpose the following questions are taken into consideration: the connection between mankind and nature, the importance of the colour blue in Goethe's studies on colour, the blue flower as a motive of the nature and Heinrich's dreams.
\end{abstract}

Keywords: Pre-Romanticism - education novel - blue - flower - fortune

Zusammenfassung: Ähnlich den Romanen Die Leiden des jungen Werthers und Wilhelm Meisters Lehrjahre von Goethe hat Novalis' Fragmentroman Heinrich von Ofterdingen die Geschichte der deutschen Literatur geprägt. Dieser Roman ist vor allem bedeutsam in der deutschen Literaturgeschichte, weil Novalis

Simone Malaguti é assistente e doutoranda na área de Literatura e Mídia do instituto de Germanistik da Universidade de Kassel. Mestre em Literatura e Língua Alemã pela FFLCH-USP. 
eine „blaue Blume“ in den Roman eingefügt hat. Dieser Artikel untersucht die Bedeutung der blauen Blume in Novalis' Heinrich von Ofterdingen als Symbol für die Suche nach dem Glück in der deutschen Literatur. Dafür werden die folgenden Fragen behandelt: die Verbindung zwischen dem Menschen und der Natur, die Farbe blau nach Goethes Lehre, die blaue Blume als Motiv der Natur und Heinrichs Träume.

Stichwörter: Frühromantiker - Bildungsroman - blau - Blume - Glück

Resumo: Assim como Os sofrimentos do jovem Werther e Os anos de aprendizado de Wilhelm Meister de Goethe, o romance Heinrich von Ofterdingen de Novalis é representativo para a literatura alemã. Este romance é também significativo e conhecido pela sua flor azul, um motivo usado por Novalis nesse romance. O artigo analisa a flor azul como um símbolo da busca pela felicidade na literatura alemã. Para tal, os seguintes assuntos são levados em consideração: a conexão entre o homem e a natureza, a cor azul conforme os estudos de Goethe, a flor azul como um motivo da natureza e os sonhos de Heinrich.

Palavras-chave: Pré-romantismo - romance de formação - azul - flor sorte

\section{Einleitung}

Ähnlich den Romanen Die Leiden des jungen Werthers und Wilhelm Meisters Lehrjabre von Goethe hat Novalis Fragmentroman Heinrich von Ofterdingen die deutsche Literatur geprägt. Als Bildungsroman behandelt auch dieses Werk die Frage der Suche nach dem Glück: Die Hauptfigur zieht in die Welt, um dort ihr Glück zu finden. Angestrebt wird eine innere Harmonie und Zufriedenheit, die aus dem harmonischen Einklang zwischen der gesellschaftlichen Ordnung und dem Ich entsteht, und die der Reisende erst am Ende seines Wegs erreicht. Viele Aspekte des Romans von Novalis sind repräsentativ für die deutsche Literatur: unerfüllte Wünsche nach Erkenntnissen, dauernde Suche, Identitätskrise, Leidenschaft, Träume, sowie Reise und Wanderung als Selbsterfahrung. Aber Heinrich von Ofterdingen ist vor allem deshalb einmalig und bedeutsam in der deutschen Literaturgeschichte, weil Novalis eine „blaue Blume“ in den Roman eingefügt hat und sie oft 
im Laufe des Romans als Symbol des Glücks und Beispiel dafür, wie und wo ein junger Mensch (hier der Jüngling Heinrich) dieses Glück suchen und finden kann, thematisiert.

Sie bildet seit der deutschen Romantik einen Schwerpunkt der intuitiven Weltschau und der Bemühung um Erkenntnis der Natur. Das Symbol übernimmt Motivfunktion, indem es zum Orientierungspunkt der Suche wird und die Wanderung begründet [...] und es zielt auf die unendliche Bewusstseinserweiterung. (DAEMMERICH 1995:76) ${ }^{1}$

Die Frage nach dem Glück ist von so grundsätzlicher Bedeutung für den Menschen, dass sie immer wieder und in immer neuen Zusammenhängen gestellt wird. Hinter den verschiedenen Konzeptionen von Glück steht die grundsätzliche Frage, was Glück denn überhaupt ist. Oft ist die Antwort darauf, ist sie einmal gefunden, gleichberechtigt mit der Selbstverwirklichung des Menschen; dabei wird dann gefragt, ob Glück in Äußerlichkeiten (in Gegenständen, im eigenen Beruf, in einem anderen Mensch oder in einem bestimmten Ort) zu finden ist, oder es nur im Inneren unabhängig von Äußerlichkeiten gefühlt werden kann. Führt es dann zu der endgültigen Selbstverwirklichung oder ist das eine Utopie, eine Illusion? Novalis' blaue Blume ist eine Metapher für die Idee, dass Glück nichts Endgültiges ist, und dass es sowohl in der objektiven und realen Umgebung liegen kann, sowie in den inneren Einstellungen des Menschen. Seine Bedeutung verändert sich ständig. Entscheidend ist nicht die Fähigkeit der Menschen, das Glück im Laufe ihres Wegs zu finden, sondern es zu erfinden. Dafür ist allerdings die Erfindungskraft, im Sinne der Einbildungskraft und der Phan-

1 Vgl. Ritzenhoff 1988: 9: „die blaue Blume ist allgemein zum Symbol für die Dichtung der Romantik im Sinne einer Sehnsucht nach dem Unendlichen geworden. Novalis hat die wunderbare Blume wie auch andere Elemente wohl hauptsächlich der schon erwähnten Kyffhäuser-Sage entnommen (vgl. dazu FRÜHWALD, „Nachwort“, 235 f.). Sie erscheint auch in dem von Johann Georg Forster (1754-94) 1791 übersetzten indischen Schauspiel Sakontala, das für Novalis eine besondere Bedeutung hatte [...] und kommt als Traumbild bereits bei Jean Paul (1763-1825) in seinem Roman Die unsichtbare Loge (1793) und im Gedicht „Der Traum“ (1799) von Ludwig Tieck (1773-1853) vor.““ 
tasie, wichtig. Das wird im Roman deutlich: Heinrich lässt sich von seinem Traum von der blauen Blume in die Welt hineinführen und begegnet Künstlern. Man könnte annehmen, dass die Selbstverwirklichung eines Menschen mit dessen Fähigkeit zur Phantasie verbunden ist.

Novalis hat diese Konzeption von Glück anhand des Symbols einer blauen Blume darstellen wollen. Dadurch hat er die Parallelität zwischen den Sphären des Menschen und der Natur hergestellt, um zu zeigen, dass die Natur vollkommen ist, und dass der Mensch sie sich zum Maßstab und zur Orientierung machen kann. Die Blume ist wunderschön und sehr begehrt, genauso wie das Glück. Die Blume ist Ergebnis einer Entwicklung von einem einfachen Samen bis hin zu einem schönen, voll erblühten Gewächs. Nach der Blüte verwelkt sie. Auch das Glück hat nach Novalis ein befristetes Dasein.

Die Analysen zur Bedeutung der blauen Blume in Novalis' Heinrich von Ofterdingen zeigen allerdings, dass die Anziehungskraft dieser Symbolik weniger von der Blume an sich ausgeht, wie Hecker in seiner Arbeit (1931) suggeriert, als vielmehr von ihrem Blau als Farbe. Während sich die Blume verwandelt und ihre Anziehungskraft (für den jungen Heinrich) verliert, ist ihr Blau immer anwesend. Overath (1987) macht auf die Besonderheit der Farbe Blau in der Literatur aufmerksam. Sie beobachtet Blau-Phänomene bzw. die Wirkung dieser Farbe im Werk von verschiedenen Autoren im 19. und 20. Jh., und sie stellt fest, dass „diese Farbe die Tendenz hat, Außerliterarisches zu tangieren“ (Overath 1987: 4). Das Außerliterarische war auch das Ziel der sog. Transzendentalpoesie der deutschen Romantik, einer Literaturtheorie, deren Hauptinteresse in der Idee des unendlichen Progresses lag, den das Ich auf dem Wege zu einer höheren Totalität von Natur und Geist durchlaufe. Im Hintergrund steht der Kampf gegen den aufgeklärten Rationalismus, bzw. eine Entwicklung des Geistes, die jeden Sinn für das Geheimnisvolle und Wunderbare tötet. Daher dient die Natur für die Romantiker als Paradigma, sie ist für sie ein Ort des Magischen und des Unerwarteten. Einen weiteren magischen Ort bilden die Welt der Imagination und der Phantasie. Auch sie werden in die Poesie miteinbezogen, um Sinnlichkeit zu stiften. Wie dieser Aufsatz im Folgenden zeigen wird, wird der Rationalismus z.B. oft durch die Figur des Vaters dargestellt. Der Vater wird allerdings als Vorbild zum Glücklichsein abgelehnt. 
Die Naturauffassung hat zu Novalis' Zeit eine größere Rolle gespielt, als sie dies in der gegenwärtigen Literatur tut. Bevor spezifische Stellen des Romans untersucht werden, soll deshalb diese Naturauffassung vorgestellt werden, die für die verschiedenen Bedeutungen der blauen Blume aufschlussreich sind, sowie Goethes Farbenlehre, besonders was die Farbe Blau betrifft. Goethes Studien zu den Farben waren Novalis bekannt und wurden von ihm sehr geschätzt (MOLNÁr 1973).

\section{Die Romantik und die Verbindung zwischen Mensch und Natur}

Die Jahrzehnte der Epoche der Romantik datieren um das Jahr 1800. Ihre Vertreter sind Künstler und Künstlerinnen, die in den Jahren zwischen 1760 und 1780 geboren sind. Mit diesem Zeitabschnitt überschneiden sich auch andere Epochen: die Klassik mit Goethe und Schiller, um nur die zwei prominentesten zu nennen, Junges Deutschland und Vormärz (z. B. Heinrich Heine) sowie die sog. phantastische Strömung (z.B. E. T. A. Hoffmann). Das heißt, neben der Romantik existieren zur gleichen Zeit verschiedene literarische Strömungen. Novalis gehört zu den Frühromantikern, in der Tradition des jungen Goethe mit typischen Eigenschaften von Sturm und Drang in seiner Biographie. Er ist ein romantischer Schriftsteller par excellence: ein leidender, kränklicher Mensch mit der Neigung zur Mystik, zur Philosophie und zum Wunderbarem. Ein Mensch, der im Zwiespalt zwischen Wirklichkeit und Phantasie lebt, an Weltschmerz leidet und Züge eines Genies aufweist: Im Alter von 16 Jahren hat er schon über 300 Gedichte verfasst. Sein Romanprojekt Heinrich von Ofterdingen vollendet Novalis nicht. Das Werk liegt nur als Fragment vor, das bis heute eine besondere Rezeption verlangt; auch wegen seiner Struktur, die Zitate von alten Märchen, Sagen und Gedichten einbezieht.

Ein anderes Merkmal der Epoche der Romantik, das als Hauptmotiv (Blume) in Novalis Heinrich von Offerdingen auftaucht, ist die Auseinandersetzung mit der Natur. Die Romantik war naturphilosophisch ausgerichtet. Die Romantiker setzen sich intensiv mit der Natur auseinander, erforschen und studieren sie. Sie benutzten die Natur in ihrer Poetologie, die reich an Naturmotiven ist. Aber die Natur zeigt sich in vielen Texten der Romantik eher 
als Projektionsfläche, denn hinter den Naturerscheinungen bzw. -beschreibungen stehen poetologische Überlegungen des Autors zur Dichtkunst, zu Religion und Wissenschaft. In diesem Sinn kann man davon ausgehen, dass die Natur in der Romantik poetisiert und die Poesie mit philosophischen Überlegungen aufgeladen wurde.

Die Romantiker sprachen über die Natur nicht, wie wir es heute tun. Seit der Moderne wird die Natur als ein vom Menschen getrenntes Objekt angesehen. Sie ist ein Untersuchungsobjekt und kann durch die Wissenschaft diagnostiziert, beschrieben oder sogar kontrolliert werden. Der Umgang mit der Natur während der Romantik war anders. Durch ihre Beschreibungen und Visionen der Natur wird sie (die Natur) fast wie eine Religion betrachtet, ihre Wesen erhalten z.B. manchmal heilige und wunderbare Eigenschaften zugesprochen, oder sie werden als göttlich bezeichnet. Dadurch bieten die Romantiker einen anderen Zugang zur Natur.

Die Natur soll die perfekte und vollkommene Welt darstellen, eine göttliche Welt auf der Erde. Im Vergleich zu dieser Vollkommenheit erscheint der Mensch unvollkommen; daher hoffen die Romantiker, durch die Sprache eine Art Schlüssel zu entdecken, der ihnen einen Zugang zu ebensolcher Vollkommenheit ermöglicht.

Sie träumen und sprechen von einer idyllischen Natur oder von einer Urnatur, in der Menschen, Tiere und Pflanzen in Einheit und Harmonie leben können. Mit solchen Gedanken schaffen die Romantiker eine wunderbare, fast mystische Vorstellung von der Natur. Diese Naturbetrachtung ist nicht neu, sie beruht auf Erkenntnissen, die schon von Sagen, Mythen und von christlichen Mystikern dargelegt wurden, allerdings durch die beginnende Industrialisierung verloren gegangen zu sein scheinen.

Diese Betrachtungsweise gesteht jedem Naturelement - ob belebter Organismus oder unbelebte Materie - seinen Wert in der Welt zu. Alles ist ein einziger Organismus, jedes Wesen ist mit jedem andern verbunden, jedes Wesen stammt aus der gleichen Quelle oder gehört zu einer bestimmten Ganzheit, zur absoluten Idee der Gottheit. Diese Gottheit ist in verschiedener, veränderlicher Gestalt vorhanden, so dass jeder Organismus in sich die Eigenschaft dieser Gottheit trägt. Auf diese Weise begründen die Romantiker eine gewisse Verwandtschaft zwischen der Natur und der Menschheit, die zugleich der Grund der romantischen Naturverehrung ist. 
Doch auch die Romantiker kennen schon um 1800 eine Spaltung zwischen den beiden Bereichen des Menschen und der Natur. Sie wurden von der Idee geprägt, dass die Wahrnehmung dieser Einheit zwischen beiden Bereichen mit der Entwicklung der Zivilisation, bzw. Industrialisierung, verlernt wurde. Infolgedessen ist die Natur dem Menschen fremd geworden, und der Mensch kann sie mit all seinem Wissen nicht mehr entschlüsseln. Alle Naturerscheinungen bedeuten den Romantikern viel mehr als das, was man oberflächlich sehen kann; man muss der Natur näher kommen und gewissermaßen mit dem geistigen Auge betrachten. Sie trägt mehr Bedeutung in sich als man mit Worten beschreiben oder mit Geräten messen kann.

\section{Die Farbe Blau nach Goethe}

Johann Wolfgang von Goethe war Dichter und Naturforscher in einer Person. Alles Lebendige verstand er auch symbolisch, und die einzelnen Ereignisse waren für ihn nicht unbedeutende Erscheinungen, sondern einzelne Teile einer Reihe, einer Gesamtheit. Auch der Mensch war für Goethe in diese Reihe einzuordnen; denn Goethe zufolge ist jeder Mensch in der Lage, mit der Natur zu kommunizieren. Unsere Organe verfügen über Eigenschaften, die eine Vermittlung zwischen der Außenwelt und der menschlichen Innenwelt ermöglichen. So untersuchte Goethe auch die Farben: Er meinte, dass die Farben nur deshalb überhaupt Farben sind, weil sie sich aus anderen natürlichen Phänomenen erst im Spiel des Lichts und Nicht-Lichtes, also im Zusammenspiel von Licht und Dunkelheit ergeben, das der Mensch in den Gegenständen und Wesen der Welt erkennen kann. Farben sind deswegen keine isolierten Erscheinungen der Natur, sie entstehen durch den Austausch zwischen Natur und Menschen.

Ausgangspunkt von Goethes Farbenlehre ist die Grundtrias der Farben Blau, Gelb und Rot. Diese drei Farben werden allerdings wegen ihrer (physischen) Eigenschaften in zwei Gruppen unterteilt: die Plus- und die Minusgruppe. In der Farbenlehre Goethes geht es um das Grundprinzip dieser Gruppierung, nämlich die Polarität von Licht (Plusseite) und Nicht-Licht (Minusseite) bzw. von Hell und Dunkel.

Gelb und Rot ähneln sich, was die Länge der elektromagnetischen Welle betrifft, und gehören deshalb zusammen, während sich Blau von den 
beiden abhebt und allein steht. Der Farbton Blau liegt nach Goethe auf dem Pol Nicht-Licht, dagegen sind Gelb und Rot auf dem Pol Licht.

So wie Gelb immer ein Licht mit sich führt, so kann man sagen, daß

Blau immer etwas Dunkles mit sich führe. (GoEthe 1991: 252)

Gemäß dieser Systematisierung studierte Goethe die Farben unter dem Aspekt ihrer Wirkung auf den Menschen. Der Dichter erklärt nicht, was die Farben sind, sondern was sie beim Menschen auslösen, bzw. was sie bewirken. Auf diese Weise stellt Goethe eine Verbindung zwischen dem Naturphänomen Farbe und dem Menschen her. Dadurch verleiht er den Farben eine emotionale Qualität, die ihn wiederum dazu führt, ihre sinnlichsittlichen Charakteristiken zu beschreiben. Bevor die Physik die Farbfrequenzen entdeckte, hat Goethe schon von der Energie der Farben gesprochen und von der Vermittlung von Kälte und Wärme durch verschiedene Farben. Was speziell das Blau betrifft, so entdeckt Goethe z.B., dass also nicht nur die Ferne blau erscheint, sondern auch Blau Ferne evoziert. Wie Goethe selbst über die blaue Farbe formuliert:

Sie ist als Farbe eine Energie, allein sie steht auf der negativen Seite und ist in ihrer höchsten Reinheit gleichsam ein reizendes Nichts. Es ist etwas Widersprechendes von Reiz und Ruhe im Anblick. [...]

Das Blaue gibt uns ein Gefühl von Kälte, so wie es uns auch an Schatten erinnert. Wie es vom Schwarzen abgeleitet sei, ist uns bekannt. [...]

So sehen wir gern das Blaue an, nicht weil es auf uns dringt, sondern weil es uns nach sich zieht. (Goethe 1991: 252)

Wegen der Abwesenheit von Licht wird der Schatten bei Goethe als Schwarz beschrieben, und als Schutz vor dem Sonnenlicht wirkt er erfrischend, was eine Eigenschaft der Minus-Gruppe ist, in der die Kälte herrscht. Blau wird dieser Gruppe zugeordnet, wird daher auch als kalt und frisch empfunden, aber auch mit den Eigenschaften der Dunkelheit verbunden. Für Overath (1987: 18) liegt die Besonderheit der Farbe Blau in ihrer Verbindung von sich scheinbar Widersprechendem: Obwohl die Farbe Blau vom Dunklen kommt, ,weist sie die Reinheit, ein klares, reines, unbeflecktes Nichts auf“. 
Blau ist also nach Goethe die einsame Farbe der Trias der Grundfarben, die in sich ihre dunkle Herkunft bewahrt, doch auf ein Helleres verweist und unseren Blick so in die Ferne leitet.

Vor diesem Hintergrund der goetheschen Farbenlehre bieten sich verschiedene Interpretationen für die Farbe Blau an: Sie kann verstanden werden als ein Zeichen ferner Hoffnung, eines einzigen, aber schwer erreichbaren Ziels oder als das Erhellende, das Leuchtende in der Dunkelheit. Genauso wirkt die blaue Blume auf Heinrich: Im Traum erscheint sie ihm als eine glänzende Verheißung, als Zufluchtspunkt, als das einzige, was ihn anzieht. Um diese vielversprechende blaue Blume zu erreichen, muss er im Traum viele Hindernisse überwinden.

\section{Die blaue Blume: Ein Motiv der Natur bei Heinrich von Ofterdingen}

In erster Linie gilt die thüringische Sage von der Blume des Kyffhäusers als die Grundlage für das Motiv der blauen Blume: Ein Schäfer findet beim Weiden seiner Herde am Kyffhäuser eine wunderschöne Blume. Er pflückt sie und steckt sie an seinen Hut. Da sieht er plötzlich die Öffnung einer Höhle, deren Boden mit Schätzen bedeckt ist. Er füllt seine Taschen, und als er wieder ins Freie treten will, hört er eine Stimme, die ruft „Vergiss das Beste nicht!“ Er versteht den Sinn der Worte allerdings nicht, sucht nicht weiter sondern verlässt schnell die Höhle, die sich sofort hinter ihm schließt. Das Beste, die Wunderblume, hat der Schäfer in der Höhle verloren, wo sie für immer verschwunden blieb, ohne dass der Schäfer das merken konnte. Die wunderschöne Blume, die er unterwegs fand, war ein Zeichen an der Erdoberfläche für den versteckten Schatz, der sich in einer tieferen, unterirdischen Ebene der Natur befand. Sie ist also auch ein Zeichen für ein Mehr, das der Schäfer allerdings wegen des Reichtums am Boden der Höhle nicht behielt.

Auf der ersten Seite des fragmentarischen Romans Heinrich von Ofterdingen ist die Rede von einer blauen Blume. Sie ist das wichtigste Naturmotiv im ganzen Roman. Doch ist sie keine normale, natürliche Blume, sondern einmalig und magisch. Sie besitzt dieselbe verführerische Kraft, wie die Blume in der Sage. In ihr ist kein Schatz, sie führt dahin. Die Macht 
und Selbständigkeit der Natur im Vergleich zu dem menschlichen Bereich wird bereits deutlich, bevor der Jüngling von ihr träumt oder über die Blume spricht:

Die Eltern lagen schon und schliefen, die Wanduhr schlug ihren einförmigen Takt, vor den klappernden Fenstern sauste der Wind; abwechselnd wurde die Stube hell von dem Schimmer des Mondes. Der Jüngling lag unruhig auf seinem Lager, und gedachte des Fremden und seiner Erzählungen. ,Nicht die Schätze sind es, die ein so unaussprechliches Verlangen in mir geweckt haben', sagte er zu sich selbst; ,fern ab liegt mir alle Habsucht: aber die blaue Blume sehn ich mich zu erblicken. Sie liegt mir unaufhörlich im Sinn, und ich kann nichts anders dichten und denken. So ist mir noch nie zumute gewesen: es ist, als hätt ich vorhin geträumt, oder ich wäre in eine andere Welt hinübergeschlummert; denn in der Welt, in der ich sonst lebte, wer hätte da sich um Blumen bekümmert, und gar von einer so seltsamen Leidenschaft für eine Blume hab ich damals nie gehört. Wo eigentlich nur der Fremde herkam?’ (Novalis 1802: 9)

Die Natur mit ihrer Kraft ist im Roman immer anwesend und hat viel mehr Bedeutung als das, was sie vordergründig darstellt und als das, was man von ihr sieht. Sie ist lebendig und versucht, in das Elternhaus einzudringen. Die Eltern lassen sich von der äußerlichen Unruhe nicht stören, sie sind in einem einförmigen Schlafen versunken, also im Gleichklang mit der Wanduhr, die ein unnatürliches und maschinelles Element darstellt, während Heinrich mit der Natur im Einklang ist: auch er ist unruhig und rastlos wie der Wind draußen. Es ist, als ob Heinrich mit der Natur in Verbindung stünde und sie ihn in Bewegung halten würde. Auch er verkehrt abwechselnd in Traum und Realität, wie der Schimmer des Mondes durch das Fernster ins Haus eindringt und eine wechselnde Beleuchtung erzeugt. Es bilden sich gegensätzliche Pole:
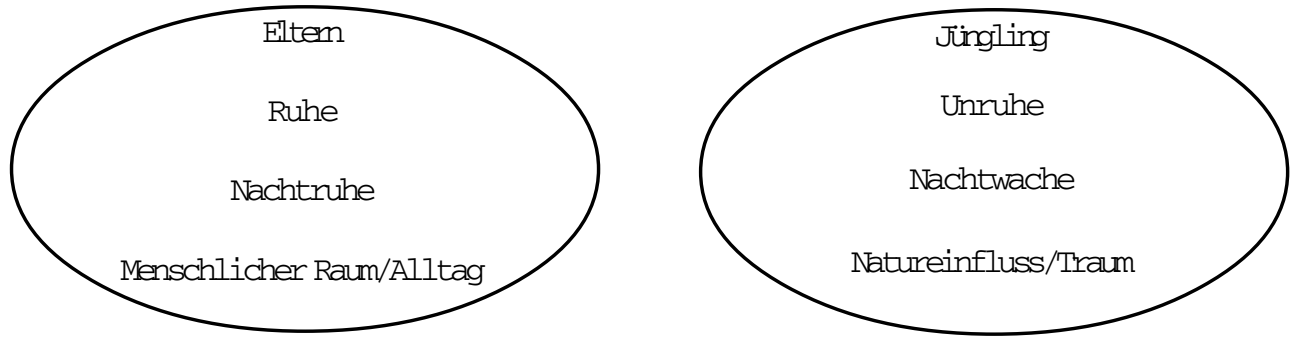
Die Eltern, das ruhige Schlafen und der Hausbereich weisen auf eine bestimmte Ordnung der äußeren Welt hin, die hier als eher ordentlich, unlebendig, eintönig oder gleichförmig geschildert wird. Diese zivilisierte Welt steht für die reale Ebene. Neben ihr existieren die anderen Ebenen, die der lebendigen und abwechslungsreichen Natur, zu der auch die Traumoder Phantasieebene gehört.

Am Anfang des obigen Zitates glaubt der Leser sich auf der realen Ebene, aber allmählich wird er in eine traumhafte Welt transponiert. Der Übergang dahin wird von Heinrich bestimmt: Er fragt sich, was in ihm vorgeht. Er beginnt, sich selbst zu erfahren, er sucht nach Antworten und nach den Gründen seiner Motivation, die Blume zu suchen. Heinrich spürt eine gewisse innere Unruhe, er muss fort, um Erfahrungen zu sammeln, um neue Erkenntnisse zu erlangen. Je mehr Heinrich sich in seinen Gedanken vertieft, desto mehr entfernt er sich von der Ebene der Realität und nähert sich einem paradiesähnlichen Traum:

Der Jüngling verlor sich allmählich in süßen Phantasien und entschlummerte. Da träumte ihm erst von unabsehlichen Fernen, und wilden, unbekannten Gegenden. Er wanderte über Meere mit unbegreiflicher Leichtigkeit; wunderliche Tiere sah er, er lebte mit mannigfaltigen Menschen [...]. Er fand sich auf einem weichen Rasen am Rande einer Quelle, die in die Luft hinausquoll und sich darin verzehren schien [...]. Der Himmel war schwarzblau und völlig rein. Was ihn aber mit voller Macht anzog, war eine hohe lichtblaue Blume, die zunächst an der Quelle stand [...]. Die Blume neigte sich nach ihm zu, und die Blütenblätter zeigten einen blauen ausgebreiteten Kragen, in welchem ein zartes Gesicht schwebte. Sein süßes Staunen wuchs mit der sonderbaren Verwandlung, als ihn plötzlich die Stimme seiner Mutter weckte. (Novalis 1802: 10-12)

Zuerst hat Heinrich nur von der Blume gehört, dann träumt er von ihr, glaubt, sie gesehen zu haben und ist von ihr fasziniert. Sie lockt ihn an, sie gibt ihm einen Impuls und wird zum alleinigen Ziel. Sie wird das Objekt seiner Suche und dient ihm als Orientierung. Sie führt ihn fort und verführt ihn gleichzeitig. 
Am Anfang des Romans ist die blaue Blume lediglich das innere Bild, das sich Heinrich vor ihr aus der Erzählung des Fremden macht. Die Suche nach dieser blauen Blume wird anfänglich als eine verführerische Kraft beschrieben, denn sie bringt ihn in die Welt der süßen Phantasie. Sie gilt für ihn als eine Vision, die er in der realen Welt verfolgt. Langsam wird die Blume ein wahrnehmbares Bild, das später mit der Suche nach einer Frau verbunden wird. Durch diese verschiedenen Transformationen gewinnt sie weitere Funktionen im Roman, z. B. die Funktion des Weiblichen als Orientierung für den jungen Mann.

Zusammenfassend zeigen uns die obigen Zitate: 1) die Entwicklung der Blume (vom fiktiven Element einer Erzählung in der Erzählung, vom imaginären Bild, von der blauen Blume im Traum bis hin zum Gesicht im Traum) sowie 2) den allmählichen Aufbau von verschiedenen Ebenen (Ebene der Imagination, der Phantasien und der Wünsche, Ebene der Nachtruhe und der Träume, Ebene der Fiktion (Erzählung) und Ebene der fiktiven Realität (Tagesablauf, wie z.B. im Elternhaus).

\section{Heinrichs Träume}

Im Roman werden verschiedene Träume von Heinrich geschildert. Sie haben umso mehr Bedeutung, weil sie sich später erfüllen. Sie enthalten etwas Wunderbares und geben Heinrich eine Vorahnung seiner Zukunft. Deshalb versucht er, seine Träume zu interpretieren. Er ist überzeugt davon, dass jeder Traum eine Entsprechung auf der Realitätsebene hat.

Der erste Traum (Zitat 2) bringt Heinrich zu unbekannten Gegenden, in denen er gern wandert. Mal ist der Weg leicht, ein anderes Mal schwierig: er muss Kämpfe bestehen, er stirbt und lebt weiter. Irgendwann während dieser Wanderungen befindet er sich auf einer Wiese und erblickt von da aus eine Höhle vor einem Bergmassiv. Er geht vorsichtig dorthin, ein bisschen ängstlich, aber schon bald findet er eine Quelle, ein Becken. Die Wände der Höhle werfen ein bläuliches Licht auf das Wasser. Am Becken entdeckt er erfreut die blaue Blume. Er nähert sich ihr und langsam verwandelt sich die Blume in ein hübsches Mädchengesicht. Die Verwandlung der Blume bereitet Heinrich eine wachsende Erregung, die plötzlich von der Stimme seiner Mutter unterbrochen wird. 
Dieser Moment des Erwachens ist wiederum ein Zwischenaugenblick, in dem man noch nicht völlig wach ist, aber auch nicht mehr ganz tief im Schlaf. Man befindet sich zwischen einem bewussten und einem unbewussten Zustand. Erst nach diesem Augenblick wird man ganz wach und löst sich von der Traumebene. Obwohl die Mutter Heinrich in dem Moment aufweckt, in dem er das Gesicht des Mädchens erblickt, macht ihm das nicht aus. Im Gegenteil empfindet er das mütterliche ,Guten Morgen' als beruhigend und erwidert ihre herzliche Umarmung. Diese Stelle, welche die Gleichzeitigkeit der Verwandlung der Blume in ein Gesicht und des Weckens durch die Mutter schildert, erlaubt interessante Interpretationen des Verhältnisses zwischen Sohn und Mutter:

Die Mutter fungiert nicht nur als Kontinuum der Bedeutung der Blume, sie ist auch die Verbindung von Heinrich zu seinem Ursprung, Mutter und Blume spielen eine ,ergänzende Rolle: Als Wegweiserin, als Orientierungspunkt, als Symbol der Geborgenheit, als Schlüssel einer Ganzheit. Diese Szene mit der Mutter weist auch auf das verlorene Paradies jedes Kindes, ein Paradies der ungestörten und sich selbst genügenden Mutter-Kind-Einheit. (FranKHAUSER 1997: 174)

Für die vorliegende Untersuchung ist diese Stelle wichtig, weil sie das Weibliche als Orientierungshilfe für Heinrich betont, das im Gegensatz zu dem männlichen Prinzip steht: Die Mutter ist die Person, die Heinrich weiter träumen lassen will, während der Vater ihn zum Handeln auffordert. Das zeigt auch folgende Stelle:

,Du Langschläfer', sagte der Vater, ,wie lange sitze ich schon hier, und feile. Ich habe deinetwegen nichts hämmern dürfen; die Mutter wollte den lieben Sohn schlafen lassen.' (Novalis 1802: 12)

Der Unterschied zwischen männlichem und weiblichem Prinzip klingt später noch an, wenn Heinrich mit dem Vater über seinen Traum zu sprechen versucht. Der nämlich hält weder viel vom Phantasieren, noch überhaupt etwas von dem Versuch, Träumen Bedeutung zuzuschreiben. Für Heinrich dagegen wirken die Träume fast wie eine Prophezeiung. Er nimmt seine Träume sehr ernst. Da die Mutter ihn schlafen lassen will, weiß sie offenbar von der Wichtigkeit der Träume. Es sieht so aus, als ob die Träume eine 
Reise nach innen für Heinrich wären. Sie sind eine Ergänzung zu der ,eintönigen, einförmigen' Welt der Realität. Sie gibt ihm die Kraft, sein eignes Schicksal zu bestimmen. In diesem Sinn ist der erste Traum von der blauen Blume als Initiationsereignis zu verstehen.

Nach dem Traum gerät Heinrich in schlechte Stimmung. Seine Mutter will mit ihm eine Reise unternehmen, denn sie will ihm weiter helfen, sein Schicksal zu finden. Sie fahren nach Augsburg, dem Geburtsort der Mutter. Der Abstand vom Elternhaus hält Heinrich gewissermaßen auch von der Welt der Realität entfernt. Während der Reise und in der Stadt treffen sie auf verschiedene Gruppen (Kaufleute, Bergleute, Musiker, Dichter), die für bestimmte Tätigkeiten stehen. Auf diesem Weg werden ihm Geschichten erzählt und Berufe vorgestellt. Bei seinem Großvater lernt er den Dichter Klingsohr und seine Tochter Mathilde kennen. Von da an fühlt sich der Jüngling nicht nur zur dichterischen Kunst hingezogen, sondern er verliebt sich auch in das Mädchen. Da Heinrich sich aber weiter von seinen Phantasien und Träumen bestimmen lässt, kann er irgendwann nicht mehr zwischen den imaginären und den realen Bildern unterscheiden: Wenn er Mathilde sieht, glaubt er das Gesicht des Mädchens im Traum wiederzuerkennen:

Ist es mir nicht zumute, wie in jenem Traum, beim Anblick der blauen Blume? Welcher sonderbare Zusammenhang ist zwischen Mathilde und dieser Blume? Jenes Gesicht, das aus dem Kelche sich mir entgegenneigte, es war Mathildes himmlisches Gesicht, und nun erinnere ich mich auch, es in jenem Buch gesehen zu haben. (Novalis 1802: 105)

Hier vermischen sich Bilder dreier Ebenen: Der wache Heinrich lebt mit den Bildern seiner verschiedenen Erfahrungen (Fiktion, Realität und Traumerscheinungen), d.h. auf der Ebene der Imagination, der Wirklichkeit und des Schlafens. Diese drei Ebenen vermischt er miteinander. Das geträumte Gesicht wird jetzt wirklich und bekommt einen Körper. Jetzt, wo Heinrich einen Ersatz auf der realen Ebene für die Blaue Blume gefunden hat, träumt er erneut. Diesmal geht es um Mathildes Tod, nämlich um das Übergehen der blauen Blume auf die Figur Mathildes, aber nicht um ihr Verschwinden:

Ein tiefer blauer Strom schimmerte aus der grünen Ebene herauf. [...] Sie winkte, sie schien ihm etwas sagen zu wollen, der Kahn schöpfte schon Wasser; doch lächelte sie mit einer unsäglichen 
Innigkeit, und sah heiter im Wirbel hinein. Auf einmal zog es sie hinunter. [...] Sie sagte ihm ein wunderbares geheimes Wort, was sein ganzes Wesen durchklang. Er wollte es wiederholen, als sein Großvater rief, und er erwachte. Er hätte sein Leben darum geben mögen, das Wort noch zu wissen. (Novalis 1802: 106-107)

Während sie im Wasser versinkt, spricht Mathilde ein Wort, ein geheimes Wort, das Heinrich nicht verstehen kann, denn sein Großvater weckt ihn in die Wirklichkeit zurück. Wieder vermischt Heinrich die Wirklichkeit mit der Traumwelt und will sich lieber mit dem Traum beschäftigen: Er will sich an das geträumte Wort noch erinnern. Jetzt ist der Junge nicht mehr auf der Suche nach der Blume oder nach einer Frau, sondern nach einem Wort. Eine Entwicklungskette von Symbolen - von der geträumten blauen Blume bis zu dem Wort - ist während Heinrichs Reise entstanden. Jedes Symbol entspricht einer Station und jede Station einem Erfahrungsbereich:

\begin{tabular}{l|l} 
Symbol Station & Erfahrungsbereich \\
\hline Blaue Blume & Natur, organische Welt \\
Traum eines Menschengesichtes & Sinnliche Welt \\
Eheschließung mit Mathilde & Begegnung mit Liebe und Kunst \\
Lüftung des Geheimnisses um & Hervorhebung der Bedeutung der Sprache \\
das Wort & Erlösung durch die Poesie
\end{tabular}

\section{Die Blume, das Blaue und die Bedeutung der Blauen Blume}

Die Romantiker gebrauchen die Blume als ästhetisch-mystisches Symbol. Blumen (in ihren drei Entwicklungsstufen) stehen in Beziehung zum Menschen und seinem Äußeren: Kindheit, Jugend, Erwachsensein und Alter des Menschen entsprechen Knospen, Aufblühen, Welken der Blume. Die Blumen tragen die Kraft der Erde in sich, weil sie sich von ihr ernähren, weil sie in der Erde ihre Wurzeln haben. Für das Christentum sind sie z.B. ein Element der Schönheit, der Vollkommenheit, und sie werden unter allen Formen der Natur für die sittlichste und die schönste gehalten:

Sie sind $[\ldots]$ die Hoffung auf kommende Frucht, ein verbliebenes Stück des verlorenen Paradies und vielleicht - soweit nicht der Mensch 
sie missbraucht - unter allen Geschöpfen am wenigsten vom

Sündenfluch getroffen. (ForstNER 1986: 180).

Nach Hecker (1931) gebrauchen die Romantiker ${ }^{2}$ die Blume auch als ästhetisch-mystisches Symbol für die ewige Fruchtbarkeit. So wie die Blume enthält die Farbe Blau auch eine Bedeutung: In der Geschichte der Farben ist Blau die Farbe des Himmels, des Heiligen und der Reinheit (BRUSATIN 2003: 65).

Im Heinrich von Ofterdingen ist die Blume blau, also von einer Farbe, die in Beziehung zum Dunklen, Lichtlosen, Unwirklichen, zur Transzendenz gesetzt wird. So steht die blaue Blume als Symbol für eine Art heiliges, rätselhaftes Geheimnis, dessen Entschlüsselung unmöglich ist. Der Suchende kann das nicht sofort erkennen, so dass er sich auf eine ewige Suche begibt, auf der er sich verlieren kann.

Als Zusammensetzung beider Worte hat der Ausdruck blaue Blume eine besondere Bedeutungstiefe, weil er auf die Vereinigung von irdischen und himmlischen Elementen weist:

$\begin{array}{ll}\text { Das Blaue } & \text { Blume } \\ \text { Himmel } & \text { Erde } \\ \text { Innerliches } & \text { Äußerliches }\end{array}$

Der Traum von der blauen Blume unterstützt Heinrichs Wanderung in die äußere Welt und ermöglicht ihm dabei einen intuitiven Blick auf die Liebe und Poesie. Als Symbol entspricht die blaue Blume auch dem Wunderbaren und der Anziehungskraft des Fernen, des Idealen, des Unerreichbaren und des Fremden.

\section{Schlusswort}

Der Fragmentroman Heinrich von Ofterdingen ist die Geschichte eines Lebens. Als solche zeigt er uns einen Menschen auf der Suche nach Selbstverwirkli-

2 Hecker (1931) untersucht die Blumesymbolik bei Tieck, Schlegel, Hölderlin, Fouqué und Brentano. 
chung und Liebe, bei der die Reise, die Wanderung, die Unterhaltungen und die Künste eine große Bedeutung haben. Alles ergänzt sich und trägt zu der Ausbildung des Individuums bei. Wie etwa Goethes Wilhelm Meister, geht es Novalis' Roman also um die Bildung eines jungen Menschen durch seine Erfahrungen in verschiedenartigen Wirklichkeitsbereichen. Bildung wird bei Novalis auch zur Lösung von unerfüllten Wünschen, denn sie enthält eine verführerische Kraft und bringt denjenigen, der sie wahrnimmt, in Bewegung. Die blaue Blume prägt Heinrichs Inneres, bedeutet unerfüllte Wünsche und funktioniert gleichzeitig als Ziel und als Wegweiser auf seiner Suche nach Selbstverwirklichung. Sie steht allerdings auch für die Entfernung, die man zurücklegen muss, um sich selbst zu verwirklichen.

In dieser Hinsicht könnte man überlegen, ob die Selbstverwirklichung mit dem Verstreichen von Zeit und einem Reifeprozess verbunden ist, etwa wie beim Aufblühen einer Blume. Blau als Zeichen der Reinheit und des Himmlischen verleiht der Blume Erlösungsqualitäten: Erreicht Heinrich die blaue Blume, wird er auch glücklich.

Der Roman, bzw. Heinrichs Suche, endet allerdings nicht dort, wo er glaubt, die blaue Blume gefunden zu haben. Damit verzögert Heinrich seine eigene Selbstverwirklichung, indem er sich ein neues Ziel setzt, nämlich die Bedeutung eines Wortes zu finden (Mathildes Wort vor dem Sterben). Er stellt sich momentan zufrieden und setzt die Suche fort. An dieser Stelle kommen wir zu den am Anfang des Aufsatzes gestellten Fragen: „Was ist Glück? Kann man es überhaupt finden?" Novalis zeigt in seinem Roman, dass die Suche nach dem Glück unendlich sein kann, denn das Ziel der Suche verändert sich immer, sobald man sich ihm nähert. Die Bedeutung der Reise und der Suche nach der blauen Blume liegt nicht im Erreichen des begehrten Gegenstandes, sondern in dem Erforschen und Verstehen der Empfindung dessen, was dieser Gegenstand (hier die Blume) in einem Individuum (hier Heinrich) bewirkt, dessen, was in einem selbst nur als Vorstellung existiert und sich noch nicht als etwas Konkretes in der Realität darstellt. Das sich verwandelnde Ziel in Novalis' Heinrich von Ofterdingen antizipiert gewissermaßen das Freudsche Diktum vom Weg, der selbst das Ziel ist. Novalis' Bildungsroman betont das Ausziehen in die Welt, nicht um dort sein Glück qu finden, sondern es zu machen, sich etwas einfallen zu lassen und alle Chancen zu nutzen, das Glück zu verwirklichen. In diesem 
Sinn ist Erfahren genauso wichtig wie die Selbstverwirklichung (wenn nicht sogar wichtiger), denn diese allein führt sozusagen zum Schatz, aber nicht zum Besten (wie die thüringische Sage). Vielleicht muss man, um das Beste zu haben, zuerst etwas von der Welt erfahren und etwas erleben. Dieser Weg wird in verschiedenen Werken der deutschen Literatur dargestellt oder thematisiert, wie z.B. Parzifal (1200-1210) des Wolfram von Eschenbach, Der grüne Heinrich (1854-1855) von Keller, Siddharta (1922) von Hesse, Felix Krull (1954) von Thomas Mann, Die neuen Leiden des jungen W. (1973) von Plenzdorf und Falsche Bewegung (1975) von Handke.

Wir können also anhand des hier untersuchten Romans und der oben zitierten Werke annehmen, dass die Suche nach Glück im Sinne eines Glückfindens am Zielpunkt vergeblich ist. Dabei handelt es sich um eine utopische Vorstellung von Glück, denn das Glück an sich findet man nie, Glück erfindet man für sich selbst.

\section{Literaturverzeichnis}

Gozthe, J. W. Die Leiden des jungen Werther. In: Goethes Werke. Band VI. München, Beck 1982.

Goethe, J. W. Zur Farbenlehre. In: Johann Wolfgang Goethe. Sämtliche Werke. Briefe, Tagebücher und Gespräche. Band 23 / 1. Wenzel, Manfred (Hrsg.) Frankfurt a. M Deutscher, Klassiker Verlag 1991.

Novalis [d.i.: Friedrich von Hardenberg]: Heinrich von Ofterdingen. Hrsg. v. Wolfang FRÜHWALD. Stuttgart: Reclam 1988. [1802]

Brusatin, Manlio. Geschichte der Farben. Berlin, Diaphanes 2003.

Daemmrich, Horst S. Themen und Motive in der Literatur: ein Handbuch. Tübingen, Basel, Francke 1995.

Frankhauser, Regula. Des Dichters Sophia. Weiblichkeitsentwürfe im Werk von Novalis. Köln, Weimar, Wien, Böhlau Verlag 1997.

Forstner, Dorothea. Die Welt der Christlichen Symbole. Innsbruck, Wien, Tyrolia 1986. 
HeCKer, Jutta. Das Symbol der blauen Blume im Zusammenhang mit der Blumensymbolik der Romantik. Jena Frommann 1931.

MolnÁr, Gèza von. Another Glance at Novalis Blue Flower. In: Euphorion, 67, 1973, 272-286.

Overath, Angelika. Das andere Blau: Zur Poetik einer Farbe im modernen Gedicht. Stuttgart, Metzler 1987.

SCHмiDT, Jochen. Goethes Faust. Erster und zweiter Teil. Grundlagen - Werk Wirkung. München, Beck 1999.

SCHÖNE, Albrecht. Götterzeichen - Liebeszauber - Satanskult. Neue Einblicke in alte Goethetexte. München, Beck 1982.

STRAsSER, Peter. Der Weg nach draußen. Skeptisches, metaphysisches und religiöses Denken. Frankfurt a. M., Suhrkamp 2000.

Watt, Ian. Mitos do individualismo moderno. Fausto, Dom Quixote, Dom Juan, Robinson Crusoe. Rio de Janeiro, Jorge Zahar 1997.

ZABKA, Thomas. "Dialektik des Bösen. Warum es in Goethes 'Walpurgisnacht' keinen Satan gibt.” In: Deutsche Vierteljahresschrift für Literaturwissenschaft und Geistesgeschichte 72, 1998, 201-226. 